\title{
AMYOPLASIA CONGENITA
}

(Multiple congenital articular rigidity : Arthrogryposis multiplex congenita)

BY

WILFRID SHELDON, M.D., M.R.C.P.,

Junior Physician to the Children's Department, King's College Hospital. (From the Hospital for Sick Children, Great Ormond Street, London.)

Under the names ' multiple congenital articular rigidity' and ' arthrogryposis multiplex congenita' a rare but well-defined clinical condition has

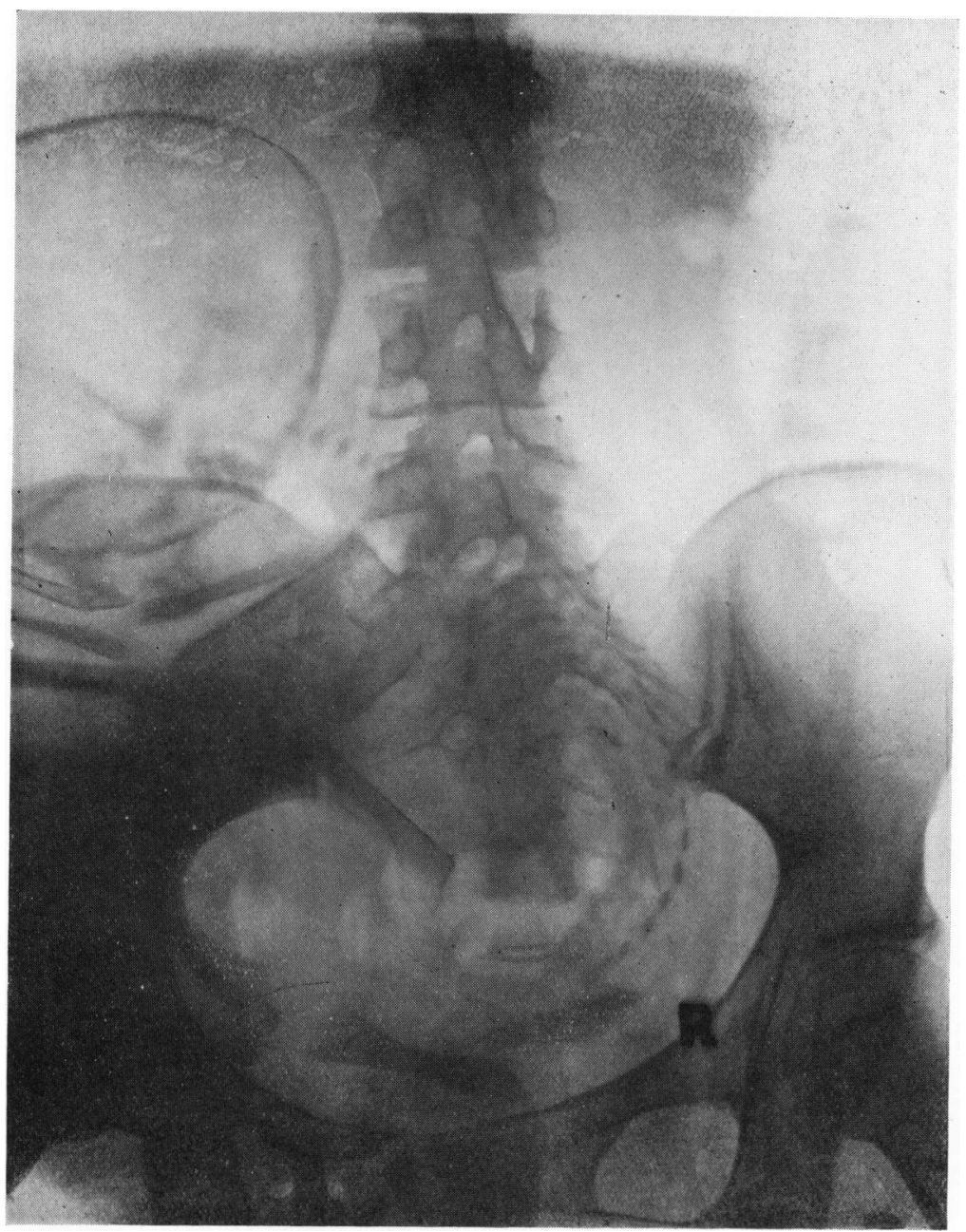

Fig. 1. X-ray of author's case in utero, showing extended position of the legs and arms. The foetal bones have been slightly retouched.

been described, the characteristic features consisting of immobility of one or more joints of the limbs, generally symmetrically distributed, and dating 
from intra-uterine life. The immobility may be absolute, or movement may be severely limited. The fixation of the joints has the clinical appearance of fibrous ankylosis, but there is no evidence of inflammatory change to account for this, and it would appear more probable that the condition depends primarily upon some developmental defect. In this connexion, the incomplete development, or even entire failure of development, of certain groups of muscles in the limbs, which has been recorded in cases specifically examined from this point of view, has been a striking feature.

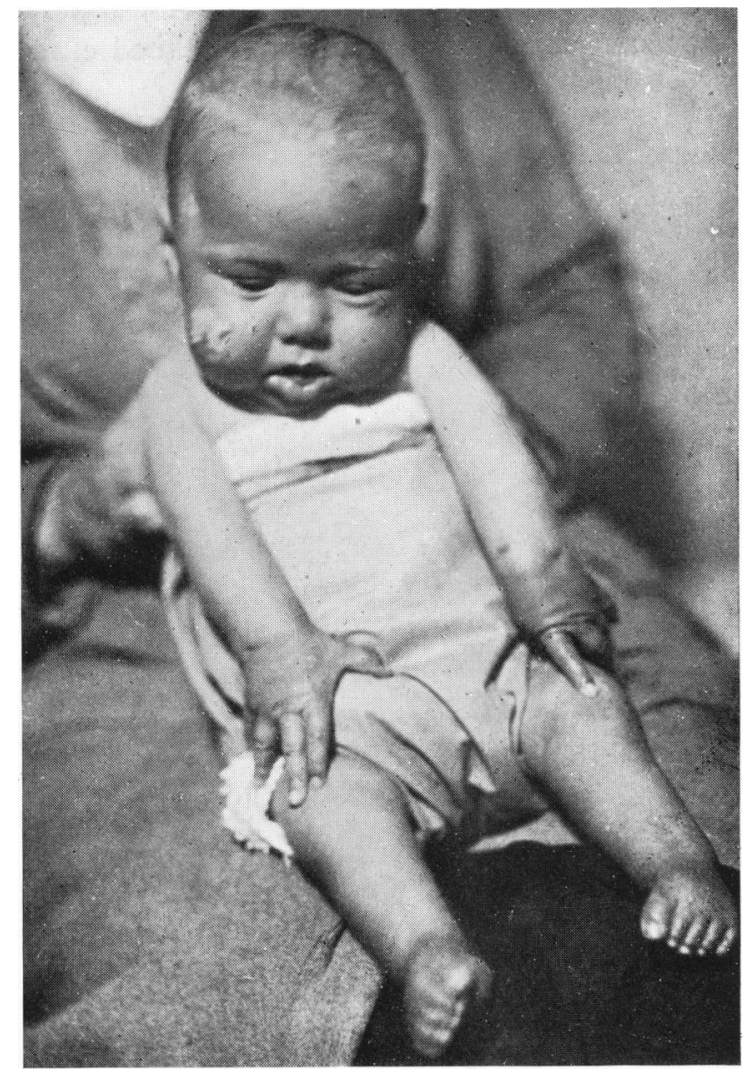

Fig. 2. Patient aged 4 months : showing the characteristic position of the arms and legs.

\section{Case report.}

The patient, a girl, is the third and last member of the family. Her parents are healthy, as are her two brothers.

The medical history of the child begins when she was a seven months fotus. She was at that time lying in a breech presentation position in utero, and an unsuccessful attempt was made under anæsthesia to turn her. An X-ray photograph of the mother's abdomen at that time (Fig. 1) shows the fœtus to be lying as a breech with extended legs, and the elbows can be seen to be extended, which is an unusual attitude. The child was born at full term as a breech with extended legs and extended arms. My thanks are due to the obstetric authorities at St. Bartholomew's Hospital for these ante-natal notes and the $\mathrm{X}$-ray photograph. 
The baby when nine weeks old was taken to the Orthopædic Department of St. Bartholomew's Hospital, and I am indebted to Mr. Elmslie for allowing me to quote the clinical condition noted at that time. The knees and elbows were rigidly extended, and could not be bent even under anæsthesia. The shoulders could be abducted to a right angle, and the wrists and fingers were held in flexion. The hip movements were fairly free. A diagnosis of ' congenital maldevelopment of joints' was made.

The child first came under the present author's care at the age of 10 weeks, when the remarkable fixation in extension of the elbows and knees was noticed (Fig. 2). Scarcely any movement was possible in these joints. Since that time the child has been under constant supervision. No treatment has been given except that carried out by the mother, which has consisted of regular daily attempts to obtain passive movements of the fixed joints. A definite improvement has occurred in both knees and to a slight extent in the right elbow, but even so the clinical features of the case remain as clear now as when first seen.

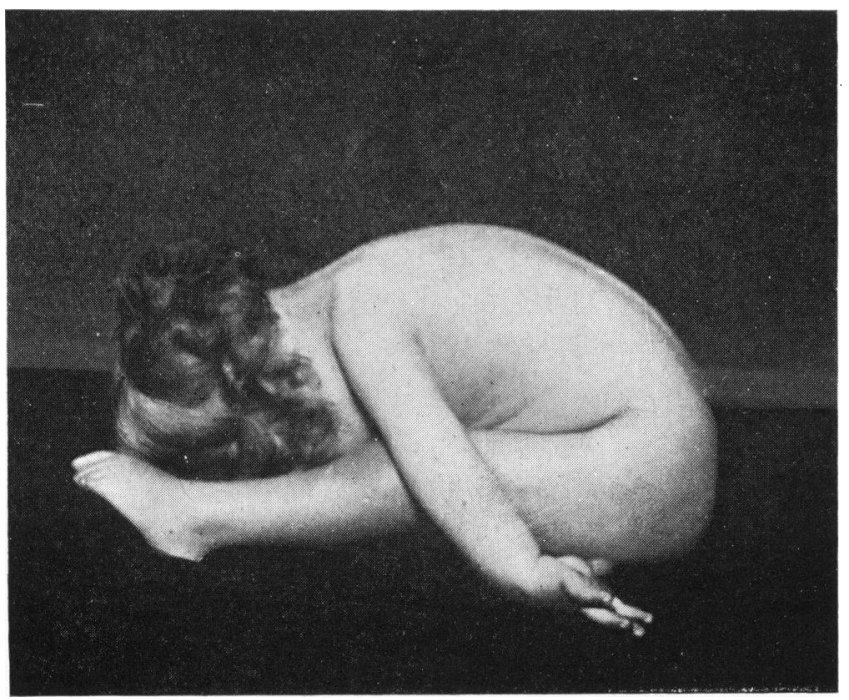

FIG. 3. Patient aged 2 years : showing the free mobility of the spine, and the rigidity of the elbow.

Examination at the present time (patient aged 2 years) shows a well nourished child, weighing $27 \mathrm{lb}$. There are three nævi, one on the cheek, one on the back, and one on the left elbow, which have been successfally treated by freezing with $\mathrm{CO}_{2}$ snow. There is nothing abnormal in the thorax or abdomen. The skull and spine are normal, and the movements of the spine and jaw are not diminished (Fig. 3). Owing to the nature of the deformities the tendon reflexes cannot be obtained, but there is no sensory loss nor trophic disturbance. Mentally the child is a normal and happy little girl, and is beginning to say short sentences.

UPPER LIMBS.-The principle features of the upper limbs are that the elbows are constantly held in rigid extension, the forearms are fully pronated, and the wrists flexed.

At the shoulders all movements can be performed, but abduction is carried out slowly and with obvious difficulty. Much of the shoulder movement depends upon the mobility of the scapula, and when this bone is prevented from moving, abduction of the shoulder is only possible to about 45 degrees, 
At the elbows the child cannot herself perform any movements, but passively both joints can be flexed through about 15 degrees. When this limit of flexion is reached, the movement suddenly checks, as happens in fibrous ankylosis. The forearm is generally fully pronated, but can be semi-supinated. Further supination is impossible. The wrists are generally flexed, but flexion is incomplete and can only be carried through about three-quarters of the normal range. The child

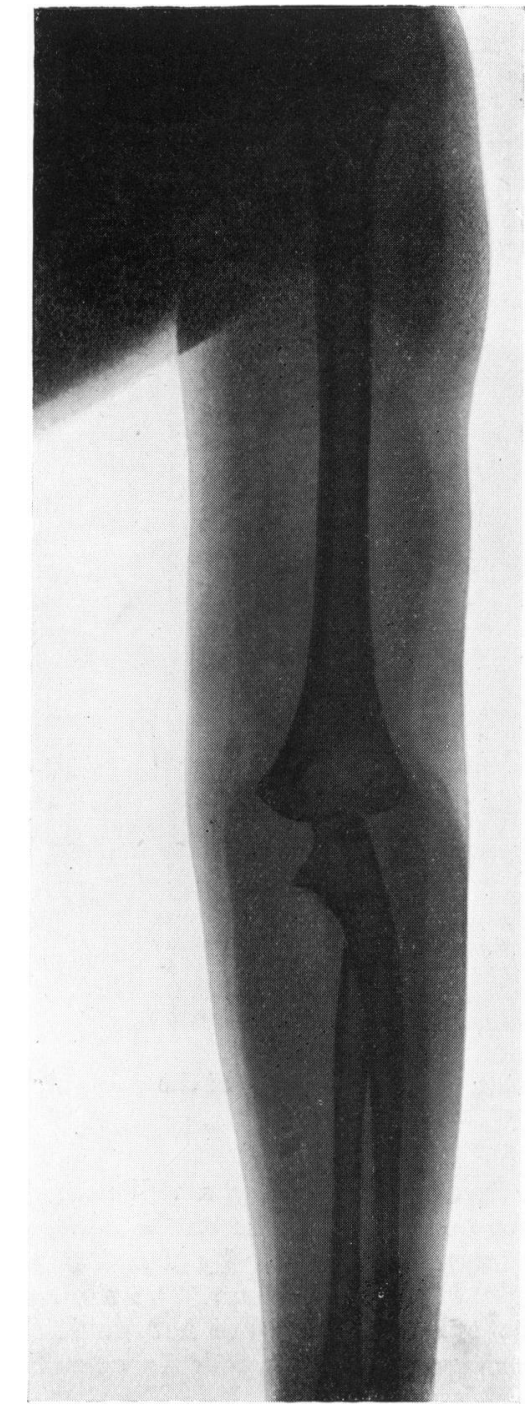

Fig. 4. X-ray of patient's arm, to show the muscle outline. The bones appear normal. Compare the muscle outline with that of a normal child (Fig. 5).

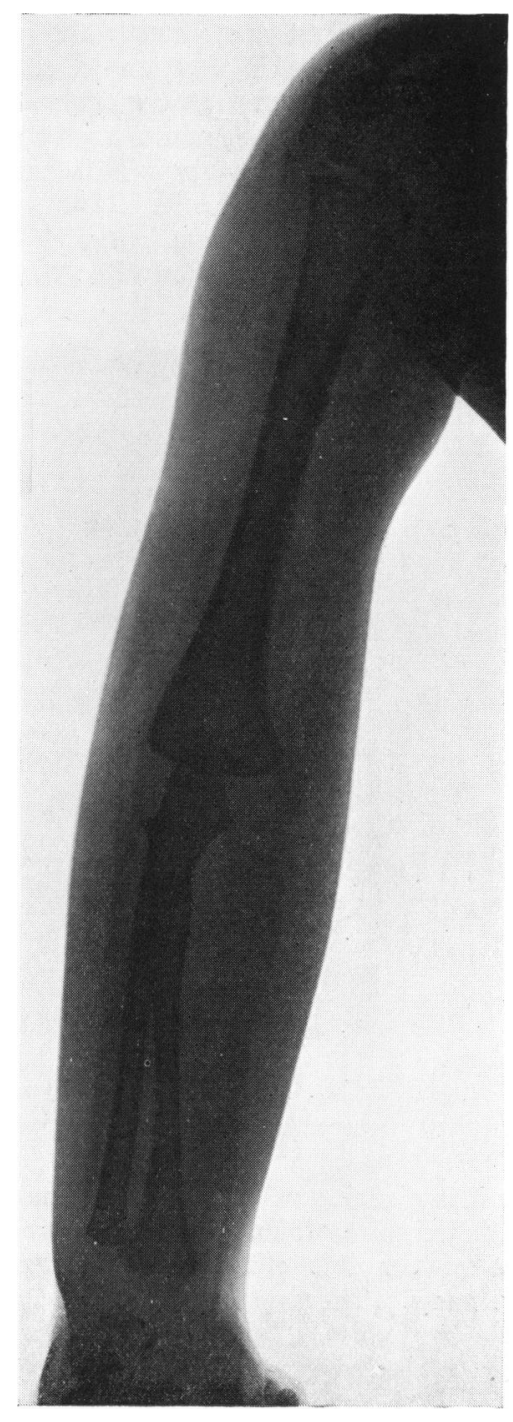

FIG. 5. X-ray of the arm of a normal child, aged 2 years (same age as patient) to show the muscle outline.

manages to extend the wrists until the hands are in a straight line with the forearms, but cannot cock the wrists. The fingers and thumbs can be moved normally.

The arms have a peculiar shape, for they taper from the wrists up to the shoulders, where the circumference of the arms is least. The appearance suggests 
that the shape of the arms is due to odema of the subcutaneous tissues, becoming more marked towards the wrists; and that some such condition may actually be present is further indicated by the absence of the normal bony contours round the elbows and wrists. There is, however, no pitting, and the nutrition of the arms as judged by their colour and warmth seems normal. There is a small dimple in the skin over the back of the styloid process of the ulna. It affects the skin only, and can be picked up and obliterated, and moved freely over the underlying structures.

X-ray examination of the bones of the upper limbs shows no abnormality of bony outline, and the bones are well calcified and appear strong. The loss of joint movement cannot be attributed to deformity of the bones.

The muscular condition of the arms has been investigated by taking ' soft' $\mathrm{X}$-ray photographs, which indicate clearly the muscular masses, and also by testing the electrical reactions. The X-ray appearance of the arm muscles is shown in Fig. 4, which should be compared with Fig. 5 showing a similar picture of the arm muscles of a normal child of sine same age. The deltoid muscle of the patient is greatly diminished, appearing like a thin strap overlying the shoulder joint. The X-ray opacity of the muscles surrounding the shaft of the humierus is also much diminished and the breadth of the muscle shadow is reduced, but in the $\mathrm{X}$-ray the triceps and biceps cannot be distinguished clearly from each other. The muscle opacity in the patient's forearm compares favourably with that of the control.

The electrical reactions to faradism were kindly tested for me by Dr. Clayton at King's College Hospital, and the results from the arm muscles are set out in Table 1 (p. 135). The following muscles gave no response at all :-the biceps, supinator longus, and extensor carpi radialis longior; the reaction of the deltoid was very weak. It may be pointed out here that the muscles which are affected are precisely those that should produce the movements which the child is unable to perform. The muscles which give a normal faradic response are those which, if actine unopposed, would place the limb in the position in which we find it; these muscles do not show hypertrophy or hypertonus so that the position of fixation of the joints, while due perhaps to the unopposed action of these muscles, is not attributable to any muscular overaction. Indeed it is likely that these unopposed muscles have, owing to lack of antagonistic action, undergone some secondary atrophy.

Lower LIMBS. - The lower limbs are maintained in an attitude of rigid extension of the knees, with bilateral talipes equinus.

The buttocks are firm and the gluteal muscles well developed. All the hip movements are easily carried out through a full range. At the knees the range of movement has increased since birth, at which time the joints were rigidly extended and flexion completely impossible. At the present time the right knee can be flexed to a right angle, and the left knee moved through about three-quarters of a right angle. The amount of active movement in the knees is a little less than can be obtained passively. During movements some fine creaking can be felt in both joints. When the limit of flexion has been reached, movement is suddenly checked as in a fibrous ankylosis.

Movement at the ankles is considerably limite!l, and the position of talipes equinus is associated with some shortening of the tendo Achillis. The toes are not moved voluntarily, but passively th:y can be fully extended.

The contour of the legs is almost as striking as that of the arms, for they taper steadily from the upper part of the thighs to the ankles. There is no bulge of calf muscles. On the lower part of each leg is a symmetrical groove in the skin, beginning over the mesial aspect of the tibia at the junction of its lower third and upper two-thirds, and running downwards and slightly outwards across the middle of the front of the ankle to end just to the outer side of the middle of the dorsum of the foot. These grooves are only skin deep, and can be moved independently of the underlying structures. They do not correspond to the direction of the tendons 
of the anterior tibial or peroneal groups of muscles. Actually no tendons can be seen or felt crossing the front of the ankle. The appearance of odema is not so noticeable in the legs as in the arms, but the bony prominences and hollows on either side of the patellar tendon are obliterated, and the knees appear as smooth spindle shaped swellings breaking the otherwise even tapering of the legs.

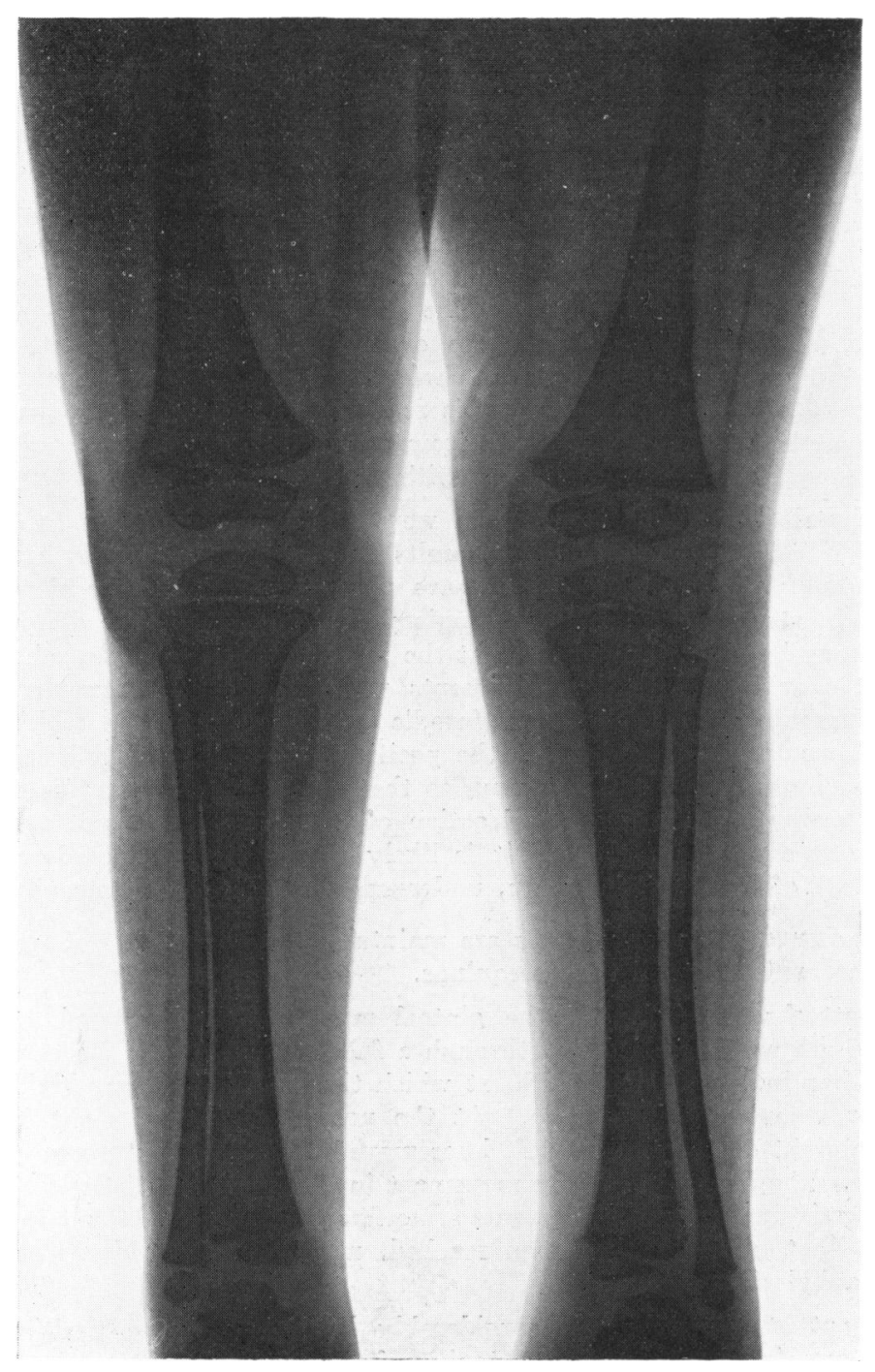

Fic. 6. X-ray of patient's legs, to show the muscle outline. The bones appear normal. Compare the muscle outline with that of a normal child (Fig. 7).

The X-ray outline of the bones of the legs is normal. There is perhaps a slight reduction in the joint space of the knees, the tibia and femur appearing a little closer together than in the normal, and the fibula seems to be displaced slightly backwards on the tibia, so that in the antero-posterior view these bones appear closer together than normal. 
X-ray investigation of the muscles of the lower limbs (Fig. 6 and 7) shows a considerable reduction in the amount of muscle surrounding the femur, and in a lateral view this deficiency is seen to affect chiefly the hamstrings. Below the knee there is nothing to be seen of the anterior tibial or peroneal groups; the gastrocnemii are reduced in size, and can be seen as a short tag on the inner side of the top of

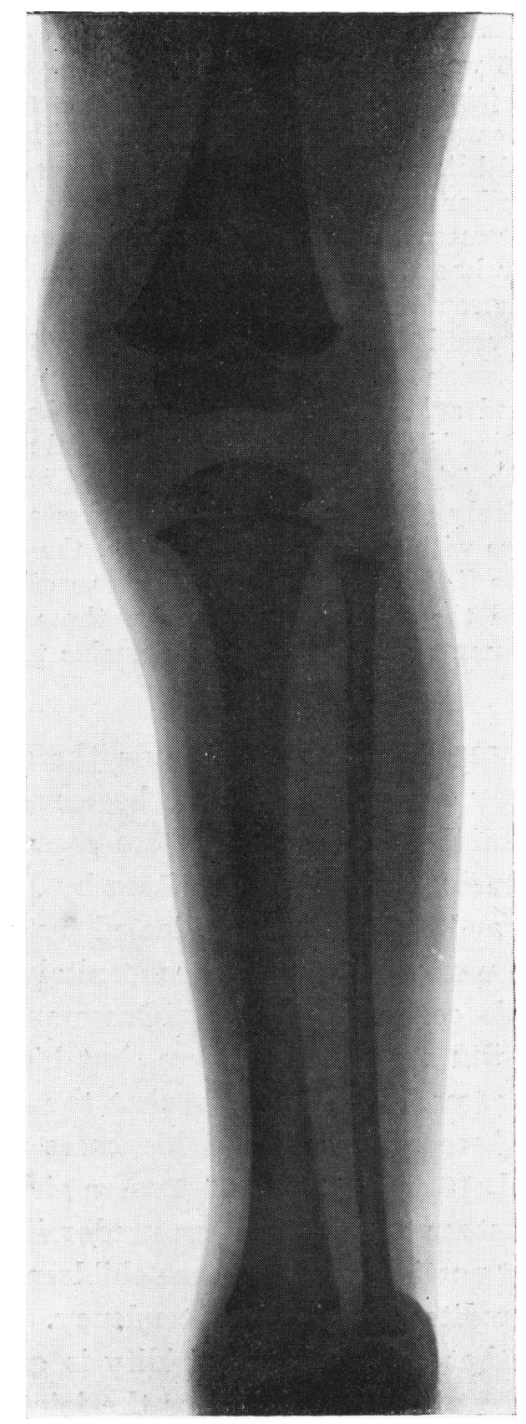

Fic. 7. X-ray of the legs of a normal child (same age as patient) to show the muscle outline (cf. Fig. 6).

the tibiæ. The capsule of the knee-joint does not appear more dense than in the normal leg.

The faradic reactions of the leg muscles are recorded in Table 2 (p. 135). The reaction from the hamstring muscles was noted as very weak, and no response was obtained from the anterior tibial or peroneal groups. As in the arm, so in the leg, the muscles which are affected are precisely those that should give rise to the 
movements which the child cannot perform. It is also curious that in the upper and lower limbs the affected muscles have corresponding functions; the flexors of the elbow and knee are either very deficient or absent, while the extensors of the wrist and ankle are also picked out.

In case there should be an element of spasm accounting for the limitation of joint movement, the child when 2 years old, was examined under an anæsthetic, but no increase of mobility was obtained. This confirmed the result of a similar examination made when the child was 6 weeks old.

The effect of the rigid joints on the child's activiti.es is severe. She is unable to feed herself in the ordinary way, and until now the mother has had to give the child all her meals. Instead of being able to crawl on her hands and knees, the child manages to move about on her feet and knuckles, the arms and legs being of course rigidly extended. At the present time she is just beginning to walk alone, making use of slight flexion of her knees. When standing, she bends forwards at the hips, counteracting this by an excessive lumbar lordosis.

Summary.-The case recorded is that of a child aged 2 years, who from birth has suffered from rigid extension of the elbows and knees, and limitation of movement in the shoulders, wrists, and ankles, inability to pronate the forearms, and bilateral talipes equinus. The condition is of prenatal origin, bilateral and strictly symmetrical. The bony skeleton and the joint contours are normal. The range of movement of the affected joints is suddenly checked, simulating fibrous ankylosis, and indicating undoubted intrinsic changes within the joints. Finally, there is great diminution of some muscle groups in the limbs, and complete absence of others. This muscular defect is symmetrical, and the affected muscles are those which should normally give rise to the very movements which the child is unable to perform.

\section{Discussion.}

Previously recorded cases.-The literature on the subject of this disease is scanty, and practically all examples have been recorded by orthopædic surgeons, to whom, as might be expected, these cases gravitate.

The most comprehensive paper is one written by Rocher ${ }^{1}$ in 1913, based on four personal cases and 26 instances collected from the literature. $\mathrm{He}$ employs the descriptive name of ' multiple congenital articular rigidities,' a name first given to this condition by Nové-Josserand. A description of Rocher's various cases will not be given here, but his clinical account and the photographs of his cases leave no doubt that the condition he describes is identical with that here recorded. Of his cases in which the sex of the child was mentioned, 15 were boys and 7 were girls, but the figures are too small to indicate whether there is any sex predominance. The condition does not appear to affect particularly first-born children, and from the family histories there is no hereditary or familial tendency.

Rocher points out that although the rigidity is generally bilateral and symmetrical, it may affect only one joint, or the joints of one limb, or the position in which fixation occurs may differ in corresponding joints of two limbs. He describes the three following clinical types:-

1. Fixation of all four limbs in extension. This is the most frequent type, and the case recorded here is a typical example.

2. Fixation of the knees in flexion, sometimes combined with fixation of the hips in flexion and abduction.

3. Fixation of one elbow in extension, and the other in flexion. In this type the legs may be fixed in extension, 
It seems likely that fixation of the joints may occur in almost any combination, both as regards the number of joints involved, and in their position.

Rocher's analysis of his cases shows that in the first clinical type the shoulder joint is likely to be stiff, and its movements limited. The elbows are fixed in extension, the wrists are stiff and extension is limited, and the fingers are commonly in the position of a 'claw-hand.' Pronation and supination can only be partially carried out, and there may be subluxation of the upper end of the radius. With regard to the muscles, the deltoid is considerably atrophied and may be absent, and the same may be true of the biceps; the triceps is on the contrary well developed. The extensor muscles of the forearm are poorly developed, while the flexors of the wrists and fingers are generally normal. Shortening of the flexor tendons of the fingers has been recorded as in Volkmann's ischæmic contracture, flexion of the fingers being more easily performed when the wrists are also flexed.

The position of the lower limbs varies with the clinical type to which the child belongs. The hips may be rigidly fixed in extension, or flexion, or flexion with abduction as in the squatting position. The attitude at the hips may be only temporary or may persist. Congenital dislocation of one or both hips has been recorded as a complication.

The knees may be fixed in flexion or extension. Fixation in extension allows of flexion taking place up to a right angle; fixation in flexion may be so severe that the feet are pressed against the buttocks. Creaking can often be felt in the knees during passive movements. The feet are generally clubbed.

With regard to the muscles of the lower limb, when the knees are fixed in extension the hamstring muscles are under-developed, while fixation of the knees in flexion is accompanied by aplasia of the quadriceps. In both positions the patella is often abnormal, being displaced, small, or even absent.

The muscles never show a reaction of degeneration, so that the muscular atrophy is probably not of nervous origin, but the reactions to faradism and galvanism are diminished or absent, indicating hypoplasia or complete aplasia of muscle. There are no sensory or trophic changes. The tendon reflexes are naturally difficult to obtain, but when present they are not increased. The normal intelligence of the children is commented upon.

Rocher has also pointed out the thickened appearance oi the subcutaneous tissues obliterating the normal bony landmarks round the knees and elbows, and states that this 'fatness' may be lymphangiectatic in origin. In a case recorded by Magnus ${ }^{2}$, this subcutaneous thickening was missing, and the muscular aplasia was so great that the child appeared to be simply skin and bone. Rocher also draws attention to the little dimples in the skin over the styloid process of the ulna or on either side of the patella. Lastly, this author states that there may be some shortening of the affected limb or segment of a limb. Of associated conditions there may be ankylosis of the mandible, and some vertebral stiffness or scoliosis. 
In 1928 Rocher $^{3}$ recorded two more cases. One was a child, 12 years old, with rigid extension of the arms and flexion of the knees: the other aged 6 months with rigid hips and knees. In 1930 the same author with Ouary ${ }^{4}$ described two additional cases. In one the arms were fixed in extension, with bilateral talipes; in the other, a girl aged 3 months, there were fixation of the legs in extension and double talipes, associated with several malformations of the lumbar vertebræ and aplasia of the sacrum.

Another case was recorded by $\mathrm{Marconi}^{5}$ in 1922, and in 1926 Scarlini $^{6}$ gave an account of three cases that had been under his care. The first of these was a girl aged 7 years with elbows fixed in extension, limitation of abduction of the shoulders, and fixed flexion of the knees. A point of interest in the history of this case was that the mother did not experience quickening until the sixth month of pregnancy and fœtal movements soon faded away entirely. The other two cases were boys aged 22 months and 7 years respectively, both of whom suffered from bilateral rigidity of the knees in full flexion. In each case the arms were unaffected. Scarlini added that the rigidity of the joints was not due to bony deformity as judged by clinical and radiological examination, but he considered musculoligamentous retraction to be the cause, and he confirmed Rocher's observation that the electrical reactions of the muscles are much diminished, without showing a reaction of degeneration.

In 1923 Stern $^{7}$ summarized four cases that had been under his care, and gave to the condition the name ' arthrogryposis multiplex congenita.' In his cases there was limitation of movements of all joints except of the mandible and spine. The limitation was typical in that movement was possible for a few degrees, and then both active and passive movements were impossible. He pointed out that there was no malformation of the joint surfaces, nor congenital contracture of muscles or tendons. One case had a congenital dislocation of the hip, and another a subluxation of the tibia. Dimpling of the skin over the elbows and patellæ was noted. He commented upon the poor development of the muscles of the limbs, but ascribed this to the lack of joint movement, and a closer enquiry into the various muscle groups was not recorded.

Two years later Lewin ${ }^{8}$ recorded two cases in babies of 6 weeks and 14 months of age. He followed Stern in employing the name of ' arthrogryposis multiplex congenita.' In his cases the arms and legs were involved, the knees being fixed in flexion and the hips in abduction, so that the legs formed a diamond-shaped figure.

In addition to these cases from the literature, brief clinical notes of four cases which have been under the care of my colleague Mr. Fairbank are presented, as they are undoubted examples of the condition. I am much indebted to Mr. Fairbank for giving me access to these cases.

(1) The first was a boy aged 3 months, in whom there was a congenital limitation of extension of the left elbow, the left hand being fixed in dorsiflexion with ulnar deviation. The right hand was fixed in palmar flexion with ulnar deviation. 
The left hip was congenitally dislocated, the left knee was rigidly extended, and the left patella was absent. There was also bilateral talipes equino-varus (Fig. 8).

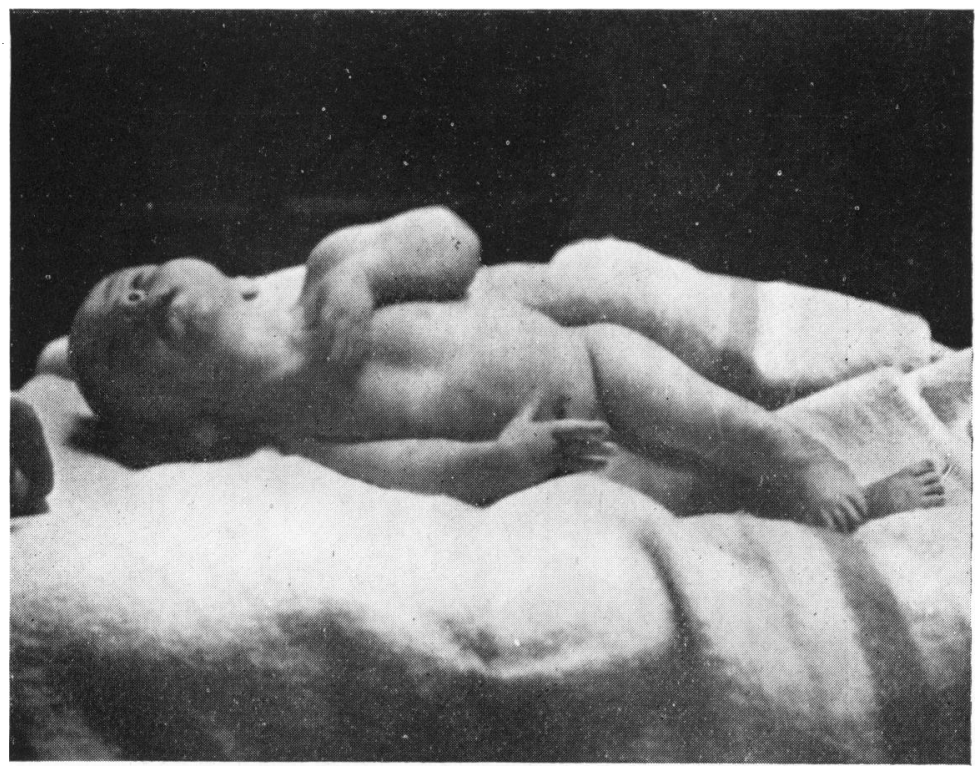

Fig. 8 .

(2) The second case was a girl aged 1 year. She was the second child of the family, and the birth had been easy. Both elbows were fixed in extension, and there was some limitation of external rotation of the humerus (Fig. 9).

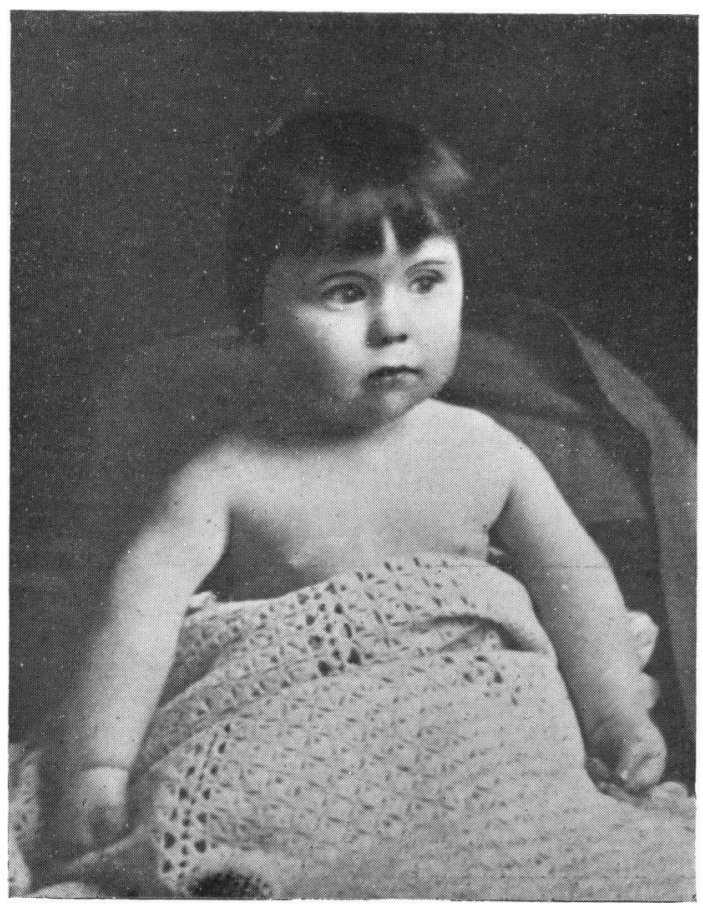

Fic: 9. 
(3) The third case was a boy aged 22 months. The elbows and knees were fixed in extension, the wrists and hands were flexed, and there was bilateral talipes equino-varus. This child also had congenital dislocation of both hips (Fig. 10).

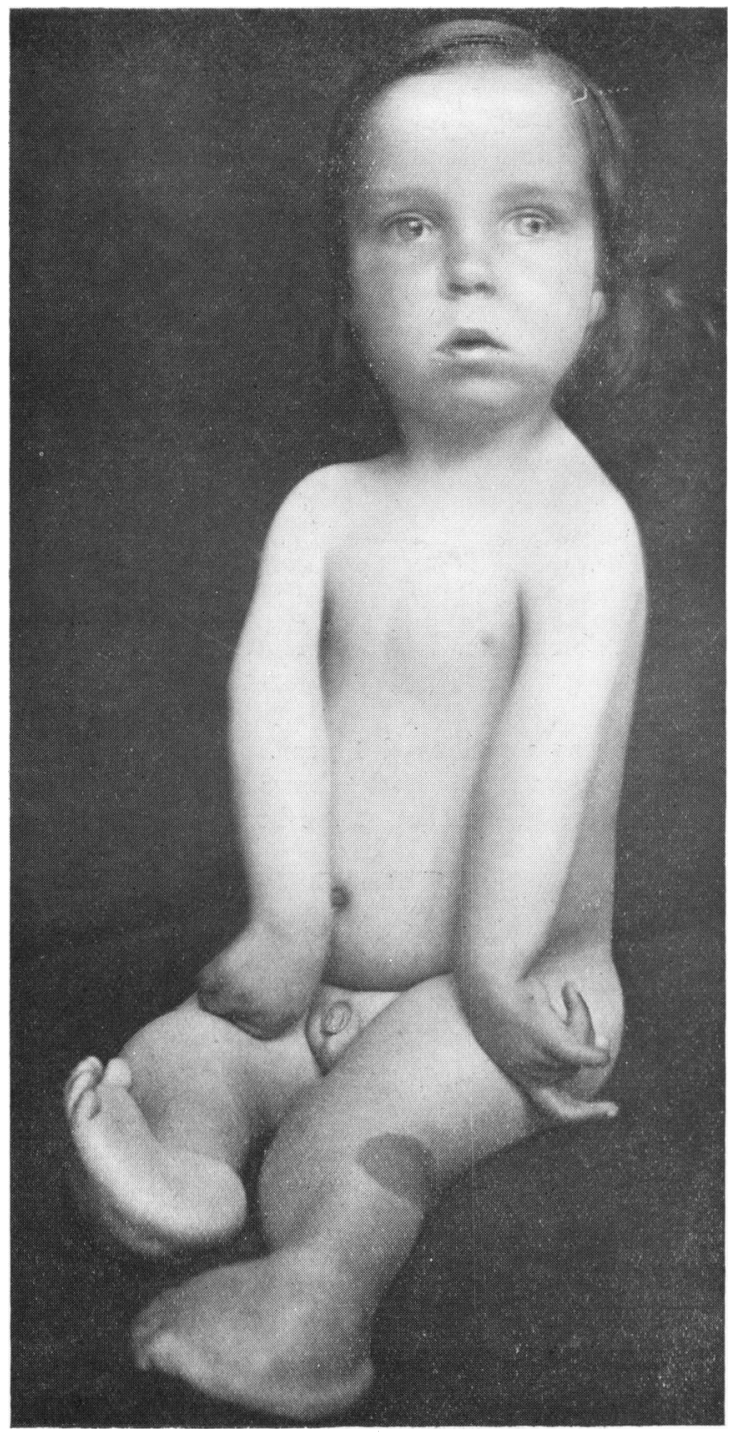

FIG. 10 .

(4) The fourth case was a boy with severe congenital talipes equino-varus, associated with flexion and abduction of the hips and flexion of the knees. At the age of 4 years, the flexion of the knees was so extreme that the feet were folded against the buttocks, extension being impossible owing to contraction of the hamstring muscles. The flexion of the knees was partly reduced by means of wedge 
plasters at a London hospital. Fig. 11 shows the condition of the legs when the boy was 6 years old, at which time he was admitted to Alton under the care of Sir Henry Gauvain. A series of corrective operations on the legs, including osteotomy

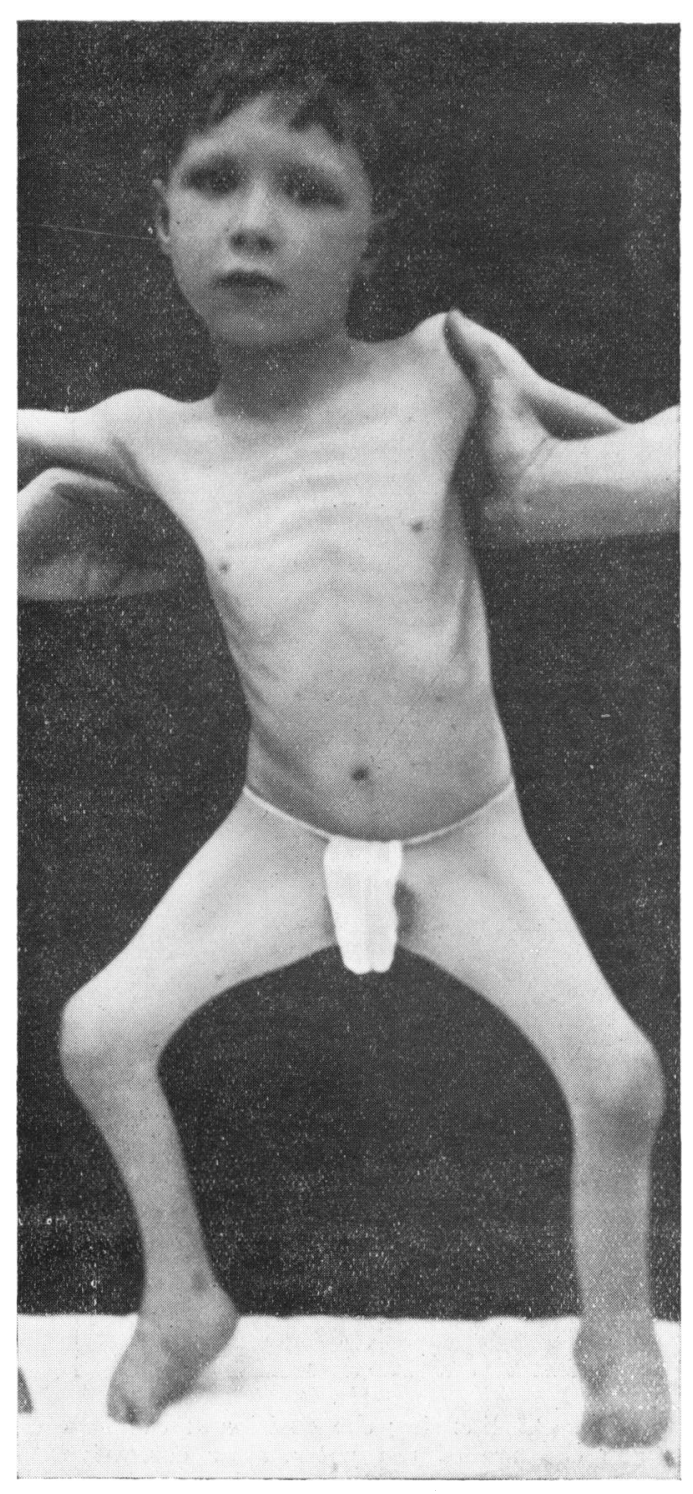

Fig. 11. Showing the position of the legs when the boy was six years old. The arms were unaffected.

of the femora and tarsal bones, was carricd out by Mr. Fairbank, and the improvement which followed is shown in Fig. 12, when the boy was 10 years old. He was then getting about well in double calipers; there was still very little movement at the hips and knees, but there was no deformity of these joints. I am much indebted to Sir Henry Gauvain for allowing me to record this case. 
A more detailed investigation of the muscles of these four children was not undertaken. These cases together with the first case recorded in this article and those already in the literature bring the total number up to 4.9 .

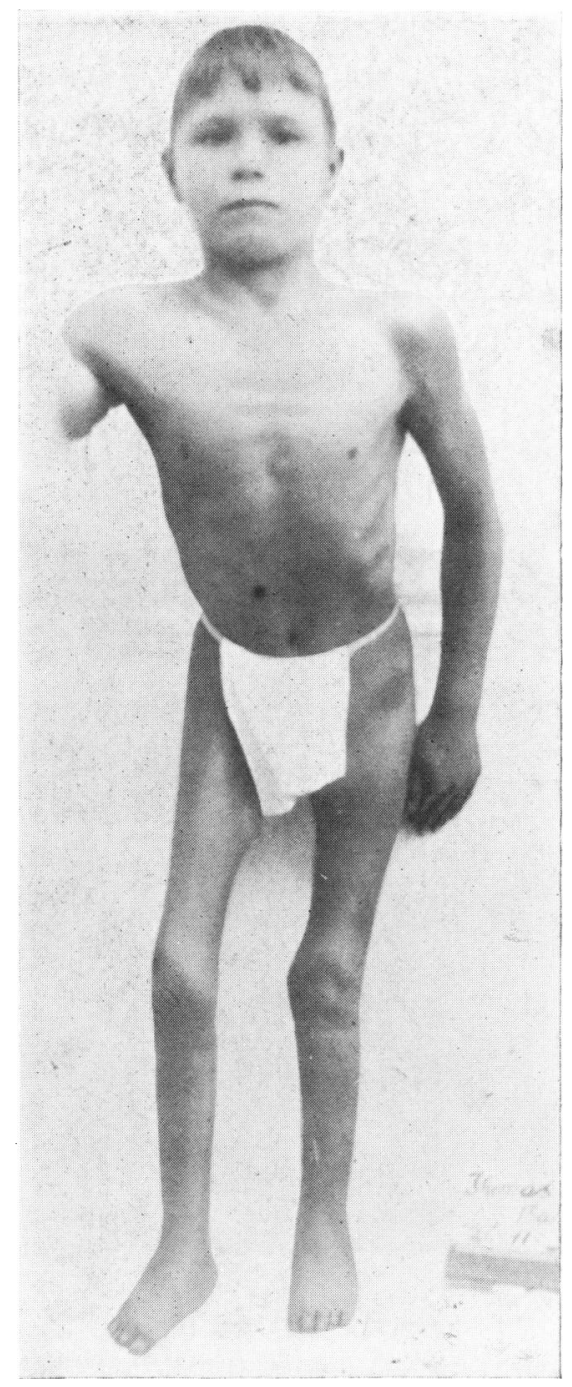

Frg. 12. Showing the position of the legs at the age of ten years, after corrective operations. Compare with Fig. 11.

Pathology.-There have been no post-mortem examinations made on this condition, and therefore our knowledge of its pathology is scanty. X-ray studies show that the bones are unaffected, except that they may take part in the general shortening of the limb which Rocher has described. Congenital subluxation and dislocation of the joints, particularly of the hip joints, is not an infrequent complication, but is either a secondary or else concomitant 
condition, since immobility also occurs when there is no dislocation. The internal anatomy of the affected joints has not been studied except that in one case of fixation of the knee it was noted that only that part of the femoral condyles which was in actual contact with the tibia was covered with cartilage; elsewhere the articular surface of the condyles was covered with fibrous tissue. Rocher states that there may be abnormal arrangement of the synovio-capsular apparatus. Congenital abnormalities of the lumbar vertebræ and aplasia of the sacrum have been reported.

Shortening of the tendons contributes to the deformity of some of the joints. In the first case recorded here, the tendo Achillis was shortened, and comment has been made upon the contraction of the flexor tendons which Rocher observed. He also adds that the skin may become contracted, as in one case of fixed flexion of the knee in which resection of the joint was performed. Before the limb could be straightened a plastic operation: on the skin behind the knee was necessary.

Under-development or even absence of certain muscle groups is indicated by clinical, radiological, and electrical studies, but few observations on the muscles have been made. Rocher states that at operation the muscles appear pale, and when cut they retract but little.

Diagnosis.-The name ' multiple congenital articular rigidity' sums up the leading clinical characteristics of the disease, and once seen the recognition of other cases should be easy. It is to be remembered that the position in which individual joints may be fixed may vary in different cases, as may the range of movement. The only other disease with which it might be confused is spastic diplegia due to lesions of the nervous system, but in that condition the stiffness of the limbs can be slowly overcome by passive means, and there is no actual fixity of the joints. Also the spasticity relaxes under anæsthesia.

Cases with fixed extension of the elbows, pronation of the forearms, and flexion of the wrists, bear a resemblance to Erb's type of brachial palsy, but in the latter condition the arms are flail, and there is no ankylosis of joints.

The rigidity is greatest at birth; it may persist undiminished, but by constant efforts at passive movements carried out over months or years, some increase in the range of mobility may be obtained. This has happened in the first case here recorded, and Stern reported that in one of his cases, passive movements led to a 25 per cent. increase of function. Major orthopædic operations have been undertaken in some of the cases with apparently good results, particularly in the cases of fixed flexion of the knees. In Scarlini's cases, osteotomy of the femur and tendon section rendered the children able to walk with the aid of splints.

Pathogenesis.-The cause of the deformities described is a matter of speculation, and it is quite likely that although these cases bear a clinical similarity to each other, more than one pathogenic process is involved. There is general agreement that the condition is present at birth, and has 
originated during intra-uterine life: also that the loss of joint movement is not the outcome of bony deformity or bony ankylosis.

Stern and Lewin attribute the condition to intra-uterine inflammation, and thickening of the periarticular tissues, with wasting of the muscles as a secondary phenomenon. However, from the history of the cases there have been no maternal illnesses which might lead to periarthritis in the fotus, and the children after birth show no interference of function of their main viscera; in fact except for their remarkable limb deformities they seem to be quite healthy. It is also difficult to regard the state of the muscles as secondary to loss of joint mobility, for the distribution of the underdeveloped museles is certainly not what would be expected to result from fixation of the joints. For instance, when the knee is fixed in extension, the quadriceps is well developed while the hamstring muscles are aplastic. Yet if in later life the knee is fixed in extension, as when the limb is put in plaster, we find that the quadriceps wastes more rapidly and to a greater extent than do the hamstring muscles. Stern also considers that the deformities are too symmetrical and regular to be attributed to abnormal embryonic growth; yet deformities of embryonic growth, such as are seen in achondroplasia or polydactyly, are usually bilateral and symmetrical. Scarlini does not deal with the ætiological aspect of the condition, which he admits is obscure, except to express his opinion that the cause does not lie primarily with the fœtus, but in some abnormality of the tissues or fluid surrounding the fœtus which prevents the normal growth of the limbs.

Rocher's view is that the initial trouble lies in abnormal compression of the fœtus, from which there follows a secondary defect of growth of all the structures which are concerned in the functioning of a joint-the bones, joint capsule, ligaments and muscles. He holds that the maldevelopment of all the tissues occurs simultaneously, and that no one tissue is primarily involved.

In the first place, what is the evidence of intra-uterine compression? Rocher lays stress on the history of oligamnios in a few of the cases. The nature of the birth is omitted in the majority of the histories, but details are obtainable in 18 cases. The labour was noted as difficult in two cases, and easy in six. Oligamnios occurred in four cases; but one of these was a fifth child, the other children being normal, although oligamnios was noted at each birth; and in another instance recorded by Scarlini the affected child was the third member of the family, the other two children being normal, and oligamnios had occurred at each birth. On the other hand, hydramnios was reported in two cases. It is difficult to see how fixation of the limbs could occur from compression in hydramnios, nor can it be presumed that unusual compression of the fotus occurred where pregnancy and labour were normal.

If abnormal compression in utero were really of ætiological importance it is surprising that its effects are confined to the limbs, leaving the vertebral and cervical movements unimpaired; torticollis might also have been expected 
to occur in some of the cases, and even evidence of pressure on the skull leading to abnormal shape, and possibly some degree of mental defect. On the whole, there seems to be no convincing evidence of abnormal compression of the fœtus in utero.

Rocher's second contention, that the defect of growth is applied simultaneously to all the structures concerned in the function of the joint, no one tissue being primarily affected, depends very largely upon the acceptance of abnormal intra-uterine compression as the origin of the condition. Such a view does not take into consideration what must surely be of significance, namely, that the aplasia of muscle is not evenly distributed among the muscles which surround the stiff joint, but picks out those by which the lost movements should be performed.

It seems likely that the peculiar distribution of the muscle aplasia is of considerable ætiological importance, and it may be that the interference with the development of the muscles is the primary lesion, to which the changes in the periarticular tissues are secondary. Some light may be thrown on this aspect of the condition by recalling the changes that take place when an ununited fracture goes on to the formation of a pseudarthrosis. In such circumstances the movements of the two fragments of bone on each other lead to a smoothing off of the bone ends, forming a shallow ball and socket joint; a joint capsule is fashioned from the surrounding fibrous tissue, while between the bone ends an adventitious bursa develops and acts as a synovial cavity. Reasoning from this, it may be supposed that in the growing limb bud of the fotus, the movements of the segments of the limb, produced by the contractions of the muscles, aid in the natural formation of the structures of a joint, and that if the muscles are unable to move the bones, the differentiation of the joint structures will be incomplete. The manner in which deformity would thus be brought about may be illustrated from the first case in this paper. In the case, for instance, of the elbow, the triceps was well developed, but the biceps group (including the brachialis anticus) was under-developed, and probably absent. With only the triceps acting, the elbow would be constantly in extension, and the structures composing the elbow joint would be correspondingly incompletely developed. The capsule would become secondarily contracted, and probably too tight to allow of complete flexion, while if scarcely any movement at all was possible, the synovial lining might either be absent or its opposite layers be adherent to each other. Secondary changes such as these at the joints would account for the clinical appearance of fibrous ankylosis. The unopposed muscles would be likely to undergo secondary atrophy and shortening.

We have as yet no knowledge of the condition of the motor nerves to the affected groups of muscles, but the fact that a group of muscles having a common function is affected rather than individual muscles is perhaps a point in favour of attributing the condition of the muscles to a primary neurone defect, and in this connection it is interesting to note that the type 
that has extended elbows and flexed wrists with limited abduction at the shoulder bears some clinical resemblance to Erb's brachial palsy due to lesions of the fifth and sixth cervical roots. On the other hand, there is no reaction of degeneration, sensation is unimpaired, and the general nutrition of the limbs is good. There is at present insufficient evidence to attribute the condition to a primary defect of the lower neurone.

The view expressed in this paper in explanation of these cases supposes that the initial defect consists of a developmental aplasia of certain groups of limb muscles. Following upon the aplasia of muscle, secondary developmental changes occur in and around the joints, leading to the clinical picture of fibrous ankylosis.

Nomenclature.-The term used by Stern and Lewin ' arthrogryposis multiplex congenita,' consisting as it does of a mixture of Latin and Greek, has little to commend it. Arthrogryposis means a bent or crooked joint, but the trouble in many of these cases is that the affected joint is persistently straight, and cannot be bent. The name also suggests that the condition is one primarily of deformed joints, and does not indicate the important changes in the muscles. Perhaps a stronger reason for rejecting this title is that ' arthrogryposis' has been used in text-books on pædiatrics both in America and in Europe as a synonym for tetany. The following passage occurs in the 1922 translation by Sedgwick and Scherer' of Feer's ' Pædiatrics ':- Tetanic carpo-pedal spasms, or arthrogryposis, consist in peculiar tonic spasms of the hand resulting in the well-known 'obstetrician's hand ',' Arthrogryposis is similarly used in Holt and Howland's ' Diseases of Infancy and Childhood '.(1926) ${ }^{10}$, and in Griffith and Mitchell's 'Diseases of Infants and Children' (1927) ${ }^{11}$.

The name ' multiple congenital articular rigidity,' used by NovéJosserand, Rocher, and Scarlini, summarizes the principal clinical features by which the condition may be recognized, but gives no indication of the important changes in the muscles. It is likely that examples of this condition have also been described under other names, such as ' multiple congenital deformities,' and ' congenital malformation of joints.'

In the belief that the initial lesion is a primary aplasia or hypoplasia of certain muscle groups, the name ' amyoplasia congenita' is put forward as conveying the origin of the condition.

\section{Summary.}

1. A case is recorded of a child with congenitally rigid arms and legs, associated with aplasia of certain muscle groups.

2. The literature of the condition is reviewed, and 44 cases are collected. In addition four cases under the care of Mr. Fairbank are added, bringing the total number of cases to 49 . 
3. The pathogenesis of the condition is discussed, and the view is put forward that the initial lesion consists of a developmental aplasia of certain muscle groups, followed by secondary developmental defects in the structure of the joints.

4. The names ' arthrogryposis multiplex congenita' and 'multiple congenital articular rigidity' are discussed, and the name 'amyoplasia congenita' is preferred.

In conclusion, I should like to acknowledge my thanks to Mr. Fairbank for his helpful criticism in the preparation of this paper.

TABLE 1.

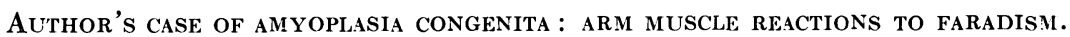

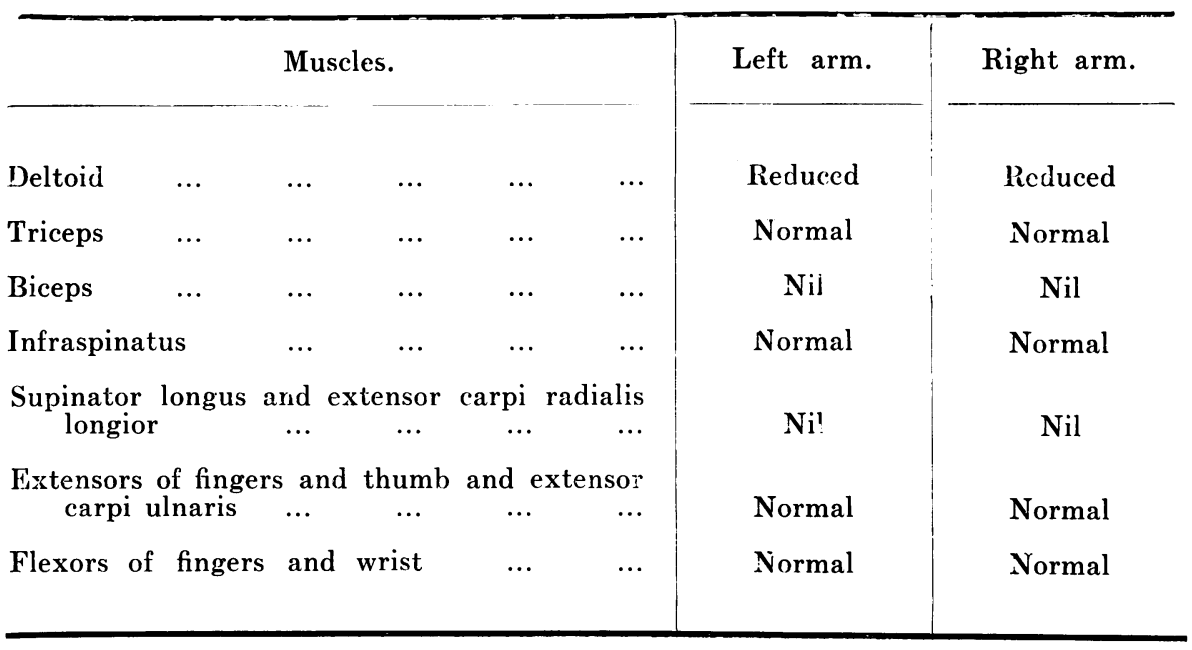

TABLE 2.

AUthor's case of amyoplasia CONGENita : Leg muscle reactions to faradism.

\begin{tabular}{lcccc|c|c}
\hline \multicolumn{3}{c|}{ Muscles. } & & & Left leg. & Right leg. \\
\hline Gluteus maximus & $\ldots$ & $\ldots$ & $\ldots$ & $\ldots$ & Normal & Normal \\
Quadriceps femoris & $\ldots$ & $\ldots$ & $\ldots$ & $\ldots$ & Normal & Normal \\
Hamstring muscles & $\ldots$ & $\ldots$ & $\ldots$ & $\ldots$ & Very weak & Very weak \\
Anterior tibial group and peroneal muscles & $\ldots$ & Nil & Nil \\
Gastrocnemius and long flexors of toes & $\ldots$ & Normal & iNormal \\
\hline
\end{tabular}




\section{REFERENCES.}

1. Rocher, H. L., J. de méd. de Bordeaux, Bordeaux, 1913, LXXXIV, 772.

2. Magnus, F., Ztschr. f. Orthop. Chir., Stuttgart, 1903, XI, 424.

3. Rocher, H. L., Congres franç. de Chir., 1928, 37th session, 1048.

4. Rocher, H. L., \& Ouary, G., Arch. franco-belge de Chir., Brussels, 1930, XXXII, 256.

5. Marconi, Arch. di Ortop., Milan, 1922, XXXVIII, 248.

6. Scarlini, G., Ibid., 1926, XLII, 451.

7. Stern, W. G., J. Am. Med. Ass., Chicago, 1923, LXXXI, 1507.

8. Lewin, P., J. Bone and Joint Surg., Boston, 1925, VII, 630.

9. Feer's Pediatrics, trans. by Sedgwick and Scherer, 1922, 536.

10. Holt and Howland's Dis. of Infancy and Childhood. 9th ed. by E. A. Part, 1926, 546.

11. Griffiths and Mitchell's Dis. of Infants and Children, 2nd ed., 1927, II, 532. 